\title{
Influence of High Fluence Nitrogen Ion Implantation on Pseudoelastic Behaviour of NiTi Shape Memory Alloy
}

\author{
N. Levintant-Zayonts* And S. Kucharski \\ Institute of Fundamental Technological Research, Polish Academy of Sciences \\ Pawińskiego 5B, 02-106 Warsaw, Poland
}

\begin{abstract}
An attempt to evaluate mechanical properties changes (superelastic phenomena) in the shape memory NiTi alloy (austenitic form) due to ion implantation $\left(\mathrm{N}^{+}\right.$, fluences of $1 \times 10^{17}$ and $4 \times 10^{18} \mathrm{~cm}^{-2}$ ) has been made. We applied the differential scanning calorimetry technique and spherical indentation (micro- and nanoindentation scale) test to study superelastic effect. The results of investigations of selected functional properties, i.e. characteristic temperatures, total and recovered penetration depth on the implanted and non-implanted NiTi samples are presented.
\end{abstract}

PACS: 62.20.fg, 81.40.Jj, 61.72.U-, 61.72.-y

\section{Introduction}

As follows from recent literature alloys with the NiTi-matrix belong to the best shape memory materials (SMAs) [1, 2]. It is well known that NiTi exhibits extraordinary shape memory and superelastic properties, which are commonly referred to as shape memory effect (SME) and pseudoelastic effect (PE). These peculiarities are a result of reversible martensitic transformation in this class of materials [3, 4]. It is well known that NiTi shape memory alloys exhibit three different phases: $B 2$ austenite (A), monoclinic $B 19^{\prime}$ martensite $(\mathrm{M})$ and trigonal $R$-phase (R) [3]. Under solution-treated conditions, near-equiatomic NiTi alloys, typically containing 49-51 at.\% $\mathrm{Ni}$, exhibit a single-stage transformation between the $B 2$ austenite (A) and the $B 19^{\prime}$ martensite (M). Under certain conditions (thermal cycling, cold working, partial annealing and ageing) the R-phase may appear between austenite and martensite. Among these three phases, several transformation sequences are possible, with various combinations of: $\mathrm{A}-\mathrm{R}, \mathrm{A}-\mathrm{M}$ and $\mathrm{R}-\mathrm{M}$ transformations $[3,4]$. All these transformations are martensitic in nature. Phase transformation from austenite (high temperature phase-stable under low stress, in a cubic form with $B 2$ symmetry) to martensite (phase-stable under adequate higher stress conditions, monoclinic form with $B 19^{\prime}$ symmetry) can be induced in SMAs above the Af temperature by loading material to exceed the critical value of transformation stress. The reverse phase transformation from martensite to austenite is observed when material is unloaded.

Unique combination of SME and PE leads to the extensive use of NiTi alloys in non-medical and medical

* corresponding author; e-mail: neonila@ippt.gov.pl applications. A wide range of their application leads to the increase of the role of their surface $[5,6]$. The issue of SMA surface modification in the context of improving its characteristics on the one hand, and maintenance of their functional properties (SME and PE) on the other hand is a subject of research and development [7, 8]. Advanced micromechanical devices need the research of micro- and nanoscale behaviour of SMAs. One of the methods used to extract small-scale mechanical properties of materials is indentation test (in micro- and nanoscales). It is known [9] that the indentation process creates high stress under indenter and can induce a phase transformation in SMAs.

The main aim of the paper is to investigate the changes of mechanical properties and a pseudoelasticity phenomenon of the shape memory $\mathrm{NiTi}$ alloy as a result of nitrogen ion implantation. We report changes of load-displacement $P-h$ curves and characteristic temperatures. We used the micro- and nanoindentation tests to observe local phase transformation and pseudoelasticity phenomena.

\section{Experimental}

The material used in this work was a commercial Ni$50.7 \% \mathrm{Ti}$ alloy (NITINOL) in the sheet form $(2.2 \mathrm{~mm}$ thickness) produced by Memory Metalle GmbH, Germany in the flat annealed form. The sheet was cut into $5 \mathrm{~mm} \times 10 \mathrm{~mm}$ flat samples. Prior to implantation, the surface of NiTi samples was mechanically polished using a polish diamond compound $(1 \mu \mathrm{m})$ and finished with the $\mathrm{Al}_{2} \mathrm{O}_{3}$ suspension. Nitrogen ion $\mathrm{N}^{+}$implantation was performed using a typical semi-industrial implanter IMJON (Institute of Fundamental Technological Research, PAS, Warsaw) with the energy $55 \mathrm{keV}$, at two fluences: $D 1=1 \times 10^{17} \mathrm{~cm}^{-2}$ and $D 2=4 \times 10^{18} \mathrm{~cm}^{-2}$. 
As the phase transformation in NiTi may be also thermally induced, differential scanning calorimetry (DSC, Pyris-1) has been used to specify the characteristic temperatures As, Af, Ms, Mf of transformation. The definitions of these temperatures are as follows: As start of austenitic phase formation in the heating process $\left(B 19^{\prime} \rightarrow B 2\right)$; Af - finish of austenitic phase formation in the heating process $\left(B 19^{\prime} \rightarrow B 2\right)$; Ms - start of martensitic phase formation in the cooling process $\left(B 2 \rightarrow B 19^{\prime}\right)$; $\mathrm{Mf}$ - finish of martensitic phase formation in the cooling process $\left(B 2 \rightarrow B 19^{\prime}\right)$.

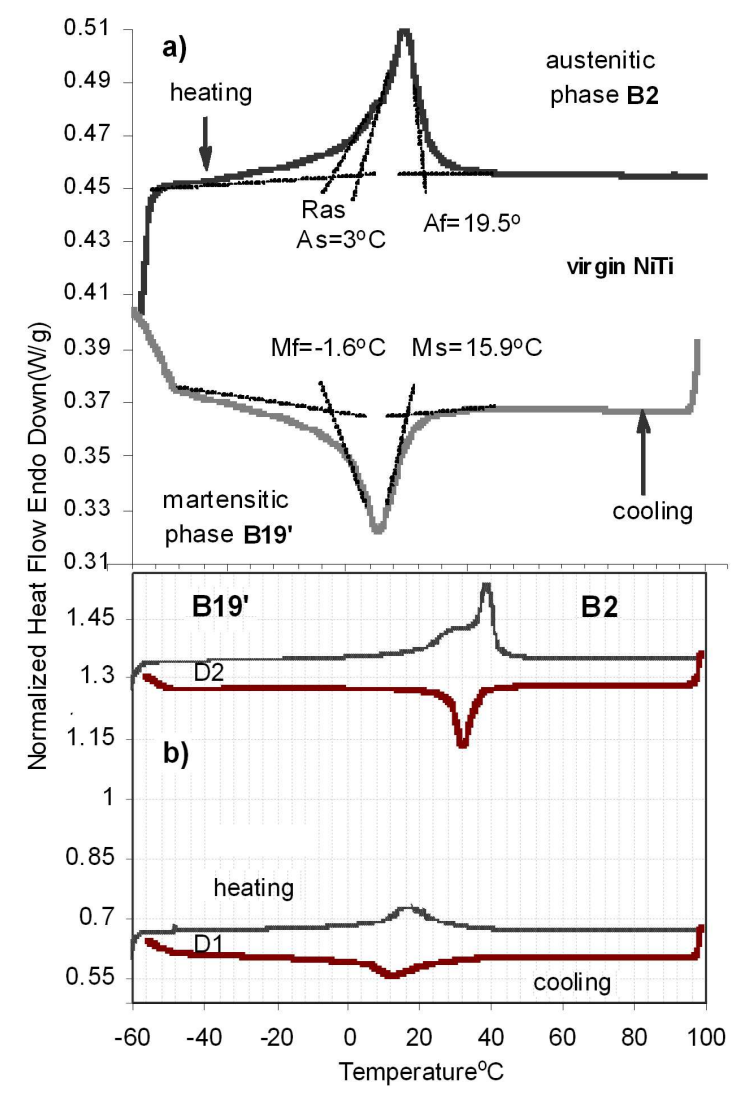

Fig. 1. Phase transformation temperatures obtained by DSC test for virgin NiTi SMA (a) and for $\mathrm{N}^{+}$implanted $\left(D 1=1 \times 10^{17} \mathrm{~cm}^{-2}, D 2=4 \times 10^{18} \mathrm{~cm}^{-2}\right)$ NiTi alloy.

The determined phase transformation temperatures of virgin NiTi: $\mathrm{Ms}=15.9^{\circ} \mathrm{C}, \mathrm{Mf}=-1.6{ }^{\circ} \mathrm{C}, \mathrm{As}=3{ }^{\circ} \mathrm{C}$, Af $=19.5^{\circ} \mathrm{C}$ are shown in Fig. 1a. At room temperature this material is in the austenitic (A) state and exhibits pseudoelastic properties. The computer program Stopping Range of Ion in Matter - SRIM 98 [10] was used to estimate the depth profile of $55 \mathrm{keV} \mathrm{N}^{+}$ions implanted into the NiTi alloy with energy of $55 \mathrm{keV}$. The effects of changing implantation parameters on the mechanical and pseudoelastic properties in the near-surface region of NiTi material were determined by using a commercially available ultra-low load indentation system, the Nano Indenter (CSM Instruments SA, Switzerland), equipped with spherical-indenter tips with different radii. In our investigations there were tested two areas of implanted NiTi samples: the part directly exposed to the ion beam irradiation, and the masked part.

The following sequences of indentation test have been made:

- microindentation tests were done by using a spherical tipped indenter $R=200 \mu \mathrm{m}$, at different loads: $4,7,10 \mathrm{~N}$;

- more shallow zone of surface was investigated using spherical indenter of a smaller radius $R=20 \mu \mathrm{m}$, at different loads: 100, 150, 200, 250, $300 \mathrm{mN}$;

- nanoindentation tests were performed using a spherical indenter $R=2 \mu \mathrm{m}$ at different loads: 0.5 and $1 \mathrm{mN}$.

All indentation experiments were performed at room temperature.

\section{Results and discussion}

This paper reports the novel application of ion implantation to modify pseudoelastic properties of NiTi alloy.

For the sample implanted with the fluence $D 1$, the calorimetric analysis (Fig. 1b) showed that during cooling from the high temperature phase, the forward transformation starts at $\mathrm{Ms}=25^{\circ} \mathrm{C}$, and ends at $5^{\circ} \mathrm{C}(\mathrm{Mf}$ - at which formation of martensite is completed). The reverse transformation starts at $\mathrm{As}=12^{\circ} \mathrm{C}$ and ends at Af $=22^{\circ} \mathrm{C}$. A similar tendency is observed for the implanted fluence D2, and the characteristic temperatures are following: $\mathrm{Ms}=36{ }^{\circ} \mathrm{C}, \mathrm{Mf}=26^{\circ} \mathrm{C}, \mathrm{As}=35^{\circ} \mathrm{C}$ and Af $=40^{\circ} \mathrm{C}$. Additionally, we can observe the appearance of the trigonal $R$-phase with $\operatorname{Ras}=25^{\circ} \mathrm{C}$ after the high dose ion implantation. After irradiation process all temperatures of martensite transformation (start/finish) are shifted to higher values. The change of transformation temperatures can be attributed to the formation of a complex structure in the near-surface region after the ion implantation, additionally - to the influence of increase in the temperature due to beam irradiation.

Micro- and nanoindentation tests were carried out on the masked (nonimplanted) and ion implanted samples. The load-displacement curves obtained in micro-indentation test (spherical indenter $R=200 \mu \mathrm{m}$ ) are shown in Fig. 2a. For both masked and implanted areas the load-unload curves $(P-h)$ exhibit hysteresis loops that correspond to the pseudoelasticity phenomena. The light stiffening of the implanted layers is observed. Very interesting indentation results were obtained for the high fluence (D2) implanted NiTi samples (Fig. 2b). The light stiffening similarly to the previous case is revealed. The occurrence of residual penetration depth on the masked and the ion implanted areas indicates that tested material does not exhibit pseudoelastic properties. However, the shape of $P-h$ curves (Fig. $2 \mathrm{~b}$ ) does not correspond to the typical character of the load-displacement 
curve for traditional elastic-plastic materials. This problem is not clearly explained. Figure 2c shows different responses to indentation of surfaces which are implanted with fluences $D 1$ and $D 2$. NiTi which was implanted with $D 2=4 \times 10^{18} \mathrm{~cm}^{-2}$ exhibits greater compliance when compared with NiTi implanted with $D 1=1 \times 10^{17} \mathrm{~cm}^{-2}$. In addition, the loop of load-displacement curve is much wider. This can be a result of "delay" of reversible transformation in the NiTi alloy. A slight decay of pseudoelasticity effect (Fig. 2c) manifested by the residual penetration depth is observed.

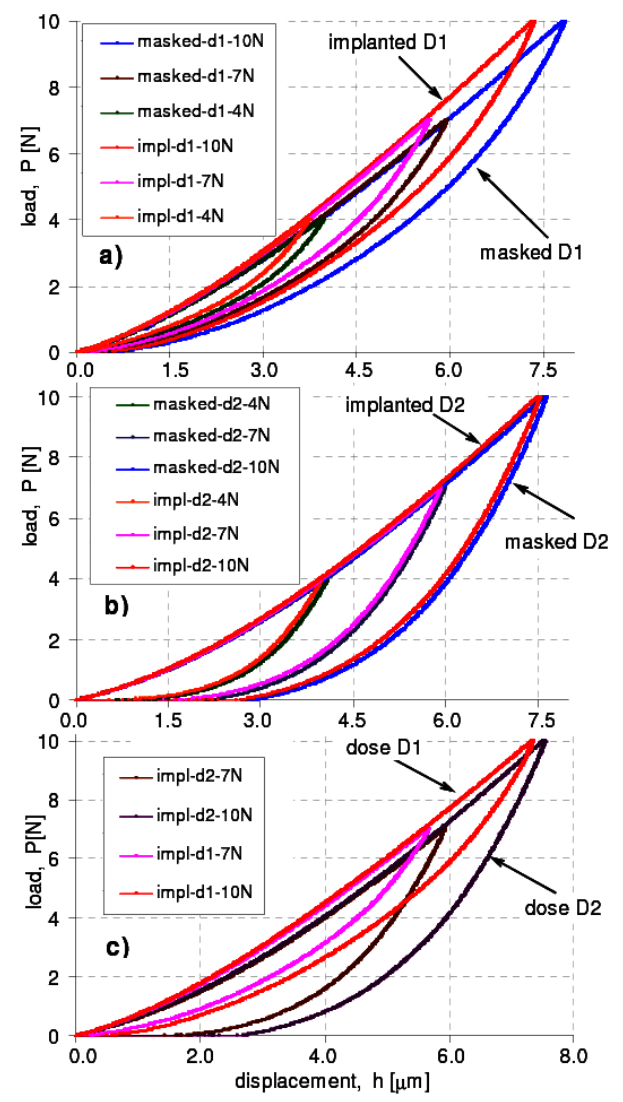

Fig. 2. Microindentation (a spherical tipped indenter $R=200 \mu \mathrm{m}) P-h$ curves for masked and ion implanted areas: with $D 1=1 \times 10^{17} \mathrm{~cm}^{-2}$ (a); with $D 2=4 \times$ $10^{18} \mathrm{~cm}^{-2}$ (b); for ion implanted with fluences $D 1$ and D2 areas of NiTi samples (c).

The load-displacement curves generated using the indenter of smaller radius $(R=20 \mu \mathrm{m})$ for the NiTi samples implanted with the fluence $D 1=1 \times 10^{17} \mathrm{~cm}^{-2}$ are shown in Fig. 3a. These curves exhibit almost pseudoelastic character for both non-implanted (masked) and ion implanted areas. Similarly to the case of the indenter $R=200 \mu \mathrm{m}$ slight strengthening of the implanted zone can be observed. Application of the spherical indenter with a smaller radius further reveals the high fluence (D2) influence, which was also observed for the microindentation test (Fig. 3b). The loop of load-displacement curve is wider. Additionally, a decay of pseudoelasticity phenomena which is manifested by the increasing value of the residual indentation depth is found.

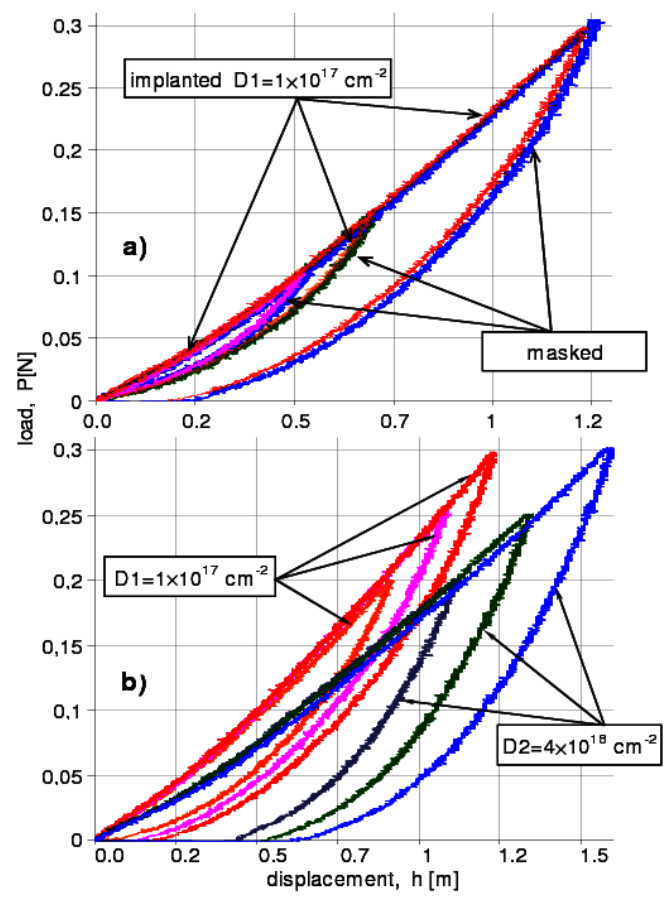

Fig. 3. Load-displacement curves received using a spherical tipped indenter $R=20 \mu \mathrm{m}$ for masked and implanted (D1) areas (a); for implanted NiTi surface at several $D 1=1 \times 10^{17} \mathrm{~cm}^{-2}$ and $D 2=4 \times 10^{18} \mathrm{~cm}^{-2}$ doses (b).

The most evident difference between the $P-h$ curves measured for implanted with fluence $D 1=1 \times 10^{17} \mathrm{~cm}^{-2}$ and masked surfaces can be observed in the nanoindentation test (Fig. 4). The implanted surface exhibits much higher stiffness and amnesia of superelasticity phenomena. There is no closed hysteresis loop. On the masked area the shape of the hysteresis loop is similar to the case of the microindentation test. The result of micro- and nanoindentation tests revealed the occurrence of larger residual depths for the implanted surface than for the masked one. This residual depth shows clearly that at room temperature the PE disappears after the high fluence (D2) ion implantation. We assume that ion implantation with a high fluence may generate modified surface layers with a gradient of defects, change of microstructure and also compressive residual stress. On the other hand, the decay of PE observed for masked (non-irradiated) areas can be a result of heating during the implantation process.

Figure 5 shows the prediction of the nitrogen ion distribution, obtained by the SRIM code. The peak of concentration value occurs at $85 \mathrm{~nm}$ for the $55 \mathrm{keV}$ implantation.

Further studies are needed to investigate the correlation between the structure of the implanted layers, its mechanical properties and conditions of the implantation 


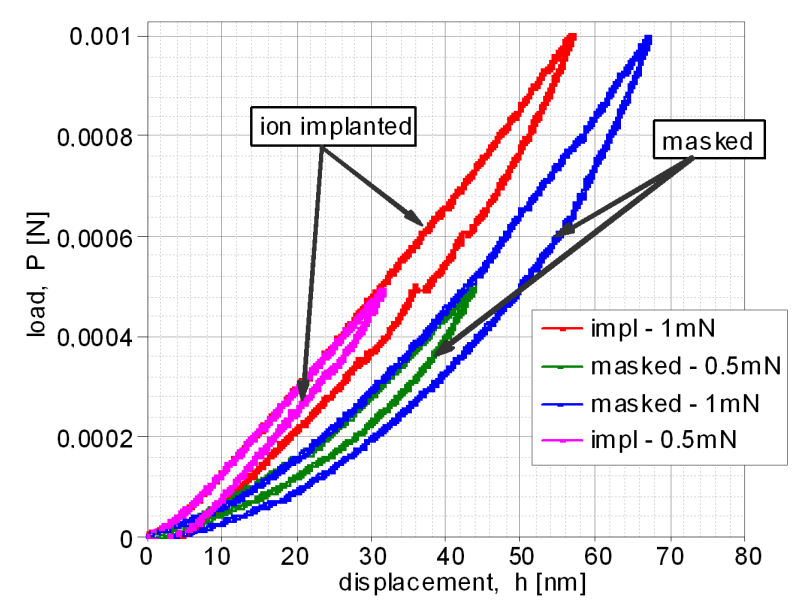

Fig. 4. Nanoindentation (spherical tipped indenter $R=2 \mu \mathrm{m}) P-h$ curves for masked and ion irradiated areas of NiTi sample, which was $\mathrm{N}^{+}$implanted with $D 1=1 \times 10^{17} \mathrm{~cm}^{-2}$.

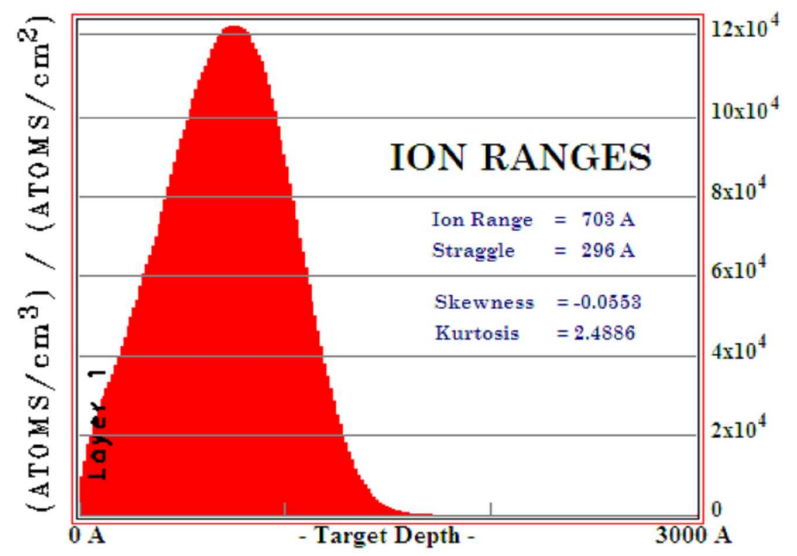

Fig. 5. SRIM-code prediction of $55 \mathrm{keV}$ nitrogen ion range distribution in NiTi target.

process.

\section{Conclusions}

The ion implanted NiTi shape memory alloy has been investigated. Our study has demonstrated the combined influence of ion implantation and heat treatment during beam irradiation on pseudoelastic properties of NiTi.

The results obtained by means of spherical microindentation, nanoindentation and DSC tests, can be summarized as follows:

- indentation of masked and ion implanted areas has shown that after the ion implantation, the "new" NiTi alloy with modified properties is obtained;
- for the low fluence of $\mathrm{N}^{+}(D 1)$ and the largest indenter radius, the pseudoelasticity effect is observed for both masked and irradiated areas;

- when the smaller volume of material (thinner layer) is investigated $(R=20 \mu \mathrm{m})$ the pseudoelasticity phenomenon is lightly disturbed, however, the stiffness of the measured areas is similar;

- in the nanoscale (60 $\mathrm{nm}$ of penetration depth) there is observed disturbance of pseudoelasticity effect in the implanted zone and maintenance in the masked region;

- the stiffness is completely different for the regions implanted with different fluences;

- all characteristic temperatures of martensite transformation (start/finish) are shifted to higher values.

These results prove that the ion implantation treatment is a suitable tool to improve mechanical properties of the near-surface layers of the NiTi alloy. The application of indentation tests can be useful in the research of thin near-surface layers of the shape memory alloys.

\section{References}

[1] L. Machado, M. Savi, J. Med. Biol. Res. 36, 3683 (2003).

[2] J. Van Humbeeck, Mater. Sci. Eng. A 273-275, 134 (1999).

[3] A. Ziolkowski, B. Raniecki, S. Miyazaki, Mater. Sci. Eng. A 378, 86 (2004).

[4] Naval Research Laboratory website: http://mstd.nrl.navy.mil .

[5] S. Shabalovskaya, J. Anderegg, J. Van Humbeeck, Acta Biomat. 4, 447 (2008).

[6] L. Tan, W.C. Crone, Acta Mater. 50, 4449 (2002).

[7] N. Levintant, Vacuum 81, 1283 (2007).

[8] W.C. Crone, H. Brock, A. Creuziger, Exp. Mech. 47, 133 (2007).

[9] C. Liu, Y. Zhao, T. Yu, Mater. Des. 26, 465 (2005).

[10] J.F. Ziegler, Particle Interaction with Matter, http://www.srim.org/. 